\title{
Um Ambiente Virtual Colaborativo para o Planejamento de Ensino PBL em Cursos de Computação
}

\author{
Jesiel Viana da Silva ${ }^{1,2}$, Gustavo Henrique da Silva Alexandre ${ }^{1}$ \\ ${ }^{1}$ Cesar School - Recife, PE - Brazil \\ ${ }^{2}$ Instituto Federal do Piauí (IFPI) - Picos, PI - Brazil \\ jesieldifpi.edu.br, gugahenrique@gmail.com
}

\begin{abstract}
Problem-Based Learning (PBL) has been a good alternative to meet the educational needs of Computing. However, there is a lack of specific digital tools to help educators in the task of PBL teaching planning. As a solution to this problem, this paper proposes the design of a collaborative system oriented to the PBL principles for teaching planning. The initial results indicate a good level of acceptance of the tool.
\end{abstract}

Resumo. Problem-Based Learning (PBL) tem sido uma boa alternativa para atender às necessidades educacionais da Computação. No entanto, faltam ferramentas digitais específicas para ajudar os educadores na tarefa de planejamento de ensino PBL. Como solução para esse problema, este artigo propõe a concepção de um sistema colaborativo orientado aos princípios PBL para o planejamento de ensino. Os resultados iniciais indicam um bom índice de aceitação da ferramenta.

\section{Introdução}

O método de ensino Problem-Based Learning ( $P B L$ ) tem se mostrado apropriado para o ensino de Computação, promovendo uma melhoria na formação profissional dos estudantes [OLIVEIRA; SANTOS; GARCIA, 2013]. PBL é um método de ensino-aprendizagem que possibilita ao aprendiz o desenvolvimento de habilidades técnicas e interpessoais integrando teoria e prática, por meio de um processo de resolução de problema [SAVERY, 2015].

Apesar dos benefícios do método PBL, estudos realizados por Santos et al. (2018), indicam que sua utilização não é simples e sua efetividade está diretamente ligada à dois fatores: aderência aos seus princípios [SANTOS; FIGUERÊDO; WANDERLEY, 2013] e a realização de um planejamento que envolva todos os aspectos e participantes do processo para que seja possível evitar que fatores críticos sejam negligenciados ou esquecidos e garantir a realização do curso na abordagem PBL com o devido alinhamento entre teoria e prática [SANTOS et al., 2018].

Nesse contexto, algumas ferramenta já foram propostas para auxiliar no planejamento de ensino PBL. [VIDAL; SANTOS; CARVALHO, 2016] desenvolveram o PBLTutor Canvas, enquanto que [ALEXANDRE.; SANTOS., 2018] criaram o PBL Planner Toolkit. As duas propostas são ferramentas visuais para o planejamento de ensino na abordagem PBL em cursos de Computação, sendo que o PBL-Tutor Canvas é específico 
para o planejamento de disciplina de forma individual e o PBL Planner Toolkit pode ser usado para o planejamento de uma ou mais disciplinas em conjunto ou curso. As duas ferramentas permitem o uso colaborativo in loco, não possuindo recursos digitais.

Embora já existam ferramentas para auxiliar no planejamento de ensino PBL, elas possuem carências que dificultam sua adoção. Algumas dessas carências é a falta de suporte à colaboração distribuída [VIDAL; SANTOS; CARVALHO, 2016; ALEXANDRE.; SANTOS., 2018], bem como a dificuldade de compartilhar exemplos de planejamentos que aumentam as chances de haver dúvidas quanto à sua utilização [ECKERSLEY; TOBIN; WINDSOR, 2018].

Diante da complexidade da implementação do PBL e das necessidades expostas acima, este artigo propõe a concepção de um sistema colaborativo aderente aos princípios PBL para o planejamento de ensino na abordagem PBL em cursos de Computação, de forma que permita a colaboração distribuída entre educadores e facilite o compartilhamento de exemplos.

\section{Metodologia}

A metodologia utilizada neste trabalho é baseada na aplicação do método Design Science Research (DSR) descrito por Wieringa (2014). Neste trabalho foram executadas as seguintes etapas do método DSR: Investigação do Problema, Projeto da Solução e Validação da Solução.

Investigação do Problema - esta etapa envolveu a busca por informações sobre o problema de pesquisa deste trabalho. Esta etapa trouxe os conhecimentos necessários para a compreensão do problema e identificação dos trabalhos relacionados.

Projeto da Solução - nesta etapa, que teve como base os resultados da etapa anterior, chegou-se a concepção da proposta de uma solução para resolver o problema identificado. Tendo em vista, que o planejamento PBL é essencialmente colaborativo, envolvendo diversos atores que trabalham de forma coletiva em objetivos comuns [BORGES et al., 2014], foi proposto o desenvolvimento de um sistema colaborativo nomeado de PBL Planner Online (PPO).

Sistemas colaborativos buscam diminuir as barreiras impostas pelo espaço físico e o tempo para promover a comunicação e ampliar a colaboração [BROUGHTON et al., 2009]. Segundo Ellis, Gibbs e Rein (1991), os sistemas colaborativos permitem que usuários interajam com outros usuários de forma comunicativa, coordenada e cooperativa para realização de tarefas com objetivo comum por meio de um ambiente virtual compartilhado. Baseado nestes conceitos, Fuks et al. (2004) apresentam o Modelo 3C de Colaboração formado pelas seguintes dimensões: comunicação, coordenação e cooperação. Este modelo foi utilizado como base para desenvolvimento do PPO.

Validação da Solução - esta etapa teve como objetivo validar a concepção do sistema, em que foi escolhido o método survey, baseado na opinião de especialistas. Para isso foi disponibilizado uma versão beta do PPO para professores acessarem e realizarem algumas tarefas pré-definidas, em seguida os participantes fizeram uma avaliação da concepção do PPO em relação ao seu objetivo, por meio de um questionário online ${ }^{1}$, denominado de Questionário de Validação.

\footnotetext{
${ }^{1}$ http://bit.ly/3aTt3hp
} 
O questionário continha 15 questões abertas sobre o perfil dos participantes e campos para comentários e 10 questões objetivas sobre cooperação, comunicação, coordenação e usabilidade, aspectos considerados importantes para concepção de um ambiente colaborativo. Para as questões objetivas, foi utilizada a escala de Likert, com escala de 1 - Discordo Totalmente até 5 - Concordo Totalmente.

\section{PBL Planner Online}

PBL Planner Online ${ }^{2}$ (PPO) é uma plataforma Web colaborativa que disponibiliza um ambiente virtual compartilhado para a realização do planejamento de ensino na abordagem PBL em cursos de Computação, com suporte à colaboração distribuída. Seu desenvolvimento foi baseado no PBL Toolkit, uma ferramenta pautada pelos princípios PBL, específica para o planejamento de ensino na abordagem PBL em cursos de Computação.

A tela de planejamento, como pode ser visto na Figura 1, é divida em três áreas: (i) uma barra no topo com o título do planejamento, listas dos usuários online e menu ações(Novo, Listar, Compartilhar e Ajuda); (ii) o Bloco de Post-its, que é um espaço na tela com um conjunto de elementos em forma de post-it, que devem ser preenchido com as informações do planejamento e movidos para os campos do PBL Canvas; e (iii) o PBL Canvas, que é um espaço de trabalho compartilhado em formato de canvas(quadro) dividido em onze campos. Cada campo é identificado por um ícone e um nome e deve ser preenchido com as informações do planejamento. As definições dos campos que formam o PBL Canvas tiveram origem nos conceitos de planejamento (PDCA) [MOEN; NORMAN, 2006] e da metodologia XPBL [SANTOS; FURTADO; LINS, 2014].

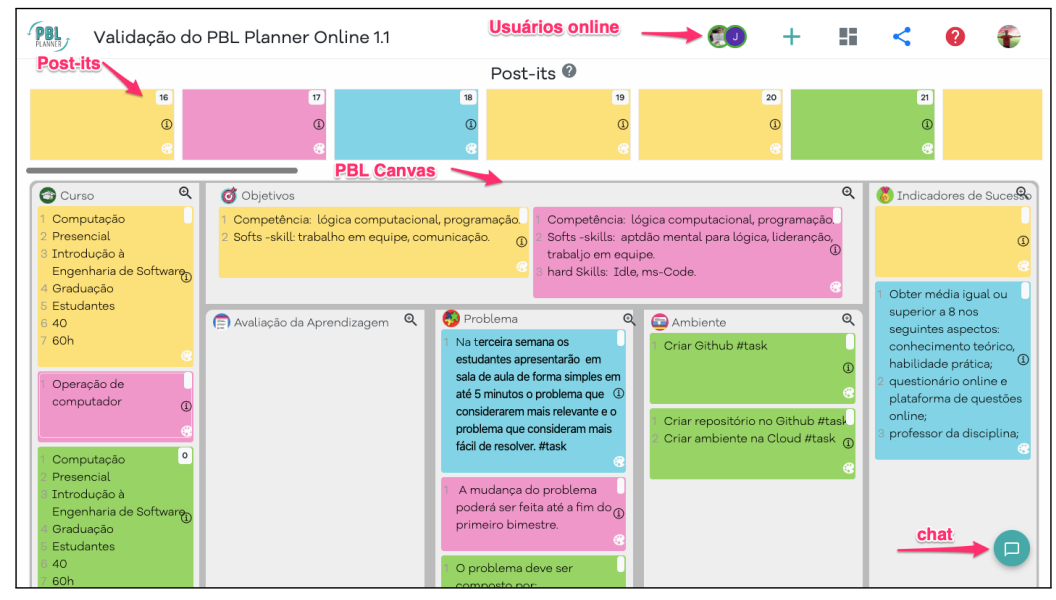

Figura 1. Tela do Planejamento

Para cada campo do PBL Canvas há um conjunto de cartas com orientação do que deve ser preenchido e exemplos prontos, que guiam o processo de preenchimento do planejamento e assim evita que os aspectos críticos do processo sejam esquecidos ou negligenciados.

\section{Resultados}

A validação da concepção do PPO contou com a participação de 9 professores de 3 instituições, sendo 3 doutorandos, 3 mestres e 3 especialistas. As respostas objetivas

\footnotetext{
${ }^{2}$ https://pblplanner.online/
} 
do questionário de validação da concepção estão consolidadas na Tabela 1.

Tabela 1. Respostas obtidas no questionário de validação da concepção

\begin{tabular}{|c|c|c|c|c|c|c|}
\hline Aspectos & Questões & $\begin{array}{l}\text { Discordo } \\
\text { Totalmente }\end{array}$ & $\begin{array}{c}\text { Discordo } \\
\text { Parcialmente }\end{array}$ & Neutro & $\begin{array}{c}\text { Concordo } \\
\text { Parcialmente }\end{array}$ & $\begin{array}{l}\text { Concordo } \\
\text { Totalmente }\end{array}$ \\
\hline \multirow{3}{*}{ Cooperação } & Q1 & & & & 1 & 8 \\
\hline & Q5 & & & & 1 & 8 \\
\hline & Q10 & & & 1 & 1 & 7 \\
\hline \multirow{3}{*}{ Comunicação } & Q2 & & & & 1 & 8 \\
\hline & Q4 & & & 2 & 1 & 6 \\
\hline & Q9 & & & & 1 & 8 \\
\hline Coordenação & Q3 & & & & & 9 \\
\hline \multirow{3}{*}{ Usabilidade } & Q6 & & & & 3 & 6 \\
\hline & Q7 & & & & 3 & 6 \\
\hline & Q8 & & & 1 & 5 & 3 \\
\hline Total Percentual & & $0,00 \%$ & $0,00 \%$ & $4,44 \%$ & $18,89 \%$ & $76,67 \%$ \\
\hline
\end{tabular}

As respostas objetivas do questionário de validação mostraram bons indicativos de que os aspectos relacionados à colaboração e usabilidade atendem às necessidades dos usuários e consequentemente à concepção da ferramenta. No total 76,67\% das respostas obtiveram pontuação máxima, indicando que os professores estão totalmente satisfeitos com os aspectos avaliados (cooperação, comunicação, coordenação e usabilidade). Os três primeiros aspectos são inerentes aos sistemas colaborativos, por isso é importante que eles atendam às necessidades dos usuários, visto que o objetivo do PPO é promover a colaboração por meio de um ambiente virtual distribuído. Já a usabilidade é um requisito essencial para qualquer sistema Web.

Por meio dos campos de comentários foi possível identificar pontos positivos do PPO na percepção dos professores, como pode ser visto nos seguintes relatos. Prof1: "A proposta é muito boa, principalmente pelo fato do planejamento ser descentralizado e poder ser feito de maneira assíncrona."; Prof2: "App promove a interdisciplinaridade entre equipes na confecção dos planos."; Prof3: "Gostei muito, pretendo utilizar nos meus planejamentos.".

Baseado na avaliação e feedback dos professores, há indicativos de que a concepção do PPO atende as expectativas de sua proposta, pois implementa um processo de planejamento guiado pelos princípios PBL, já consolidado no PBL Toolkit [ALEXANDRE.; SANTOS., 2018] e atendeu aos requisitos de usabilidade e colaboração inerentes a um sistema colaborativo.

\section{Conclusão}

Este trabalho apresentou o PBL Planner Online, uma plataforma web colaborativa, em fase de desenvolvimento, para o planejamento de ensino na abordagem PBL para cursos na área de Computação. A plataforma possui como diferencial em relação as outras ferramentas, o fato de permitir a colaboração distribuída durante a construção do planejamento alinhado com os princípios PBL e possibilitar a integração com ferramentas de gestão e monitoramento.

Como trabalhos futuros, incluem a aplicação e avaliação do PPO em ambientes reais para verificar sua contribuição ao processo de planejamento de ensino na abordagem PBL em cursos de Computação. 


\section{Referências}

ALEXANDRE., G. H.; SANTOS., S. C. Pbl planner toolkit: A canvas-based tool for planning pbl in software engineering education. In: INSTICC. Proceedings of the 10th International Conference on Computer Supported Education - Volume 2: CSEDU,. [S.1.]: SciTePress, 2018. p. 206-213. ISBN 978-989-758-291-2.

BORGES, M. de C. et al. Aprendizado baseado em problemas. Medicina (Ribeirão Preto. Online), v. 47, n. 3, p. 301-307, 2014.

BROUGHTON, M. et al. Being here: designing for distributed hands-on collaboration in blended interaction spaces. In: ACM. Proceedings of the 21 st Annual Conference of the Australian Computer-Human Interaction Special Interest Group: Design: Open 24/7. [S.1.], 2009. p. 73-80.

ECKERSLEY, B.; TOBIN, K.; WINDSOR, S. Professional experience and project-based learning as service learning. In: Educating Future Teachers: Innovative Perspectives in Professional Experience. [S.1.]: Springer, 2018. p. 175-192.

ELLIS, C. A.; GIBBS, S. J.; REIN, G. Groupware: some issues and experiences. Communications of the ACM, ACM New York, NY, USA, v. 34, n. 1, p. 39-58, 1991.

FUKS, H. et al. Applying the $3 C$ model to groupware engineering. [S.1.]: PUC Rio de Janeiro, 2004.

MOEN, R.; NORMAN, C. Evolution of the PDCA cycle. [S.1.]: Citeseer, 2006.

OLIVEIRA, A. M. C. A.; SANTOS, S. C. dos; GARCIA, V. C. Pbl in teaching computing: An overview of the last 15 years. In: IEEE. 2013 IEEE Frontiers in Education Conference (FIE). [S.1.], 2013. p. 267-272.

SANTOS, S. C. dos et al. How to apply problem-based learning in a managed way? a case in computing education. In: SPRINGER. International Conference on Computer Supported Education. [S.1.], 2018. p. 287-309.

SANTOS, S. C. dos; FIGUERÊDO, C. O.; WANDERLEY, F. Pbl-test: A model to evaluate the maturity of teaching processes in a pbl approach. In: IEEE. 2013 IEEE Frontiers in Education Conference (FIE). [S.1.], 2013. p. 595-601.

SANTOS, S. C. dos; FURTADO, F.; LINS, W. xpbl: A methodology for managing pbl when teaching computing. In: IEEE. 2014 IEEE Frontiers in Education Conference (FIE) Proceedings. [S.1.], 2014. p. 1-8.

SAVERY, J. R. Overview of problem-based learning: Definitions and distinctions. Essential readings in problem-based learning: Exploring and extending the legacy of Howard S. Barrows, v. 9, p. 5-15, 2015.

VIDAL, T. C.; SANTOS, S. C. D.; CARVALHO, R. S. Pbl-tutor canvas: A tool based on backward design to plan pbl in computing education. In: IEEE. 2016 IEEE Frontiers in Education Conference (FIE). [S.1.], 2016. p. 1-8.

WIERINGA, R. J. Design science methodology for information systems and software engineering. [S.1.]: Springer, 2014. 